\title{
Timing jitter characterization of a high-energy sub-cycle optical waveform synthesizer
}

\author{
Roland Mainz, ${ }^{1,2}$ Giulio Maria Rossi, ${ }^{1,2}$ Cristian Manzoni, ${ }^{5}$ Giovanni Cirmi, ${ }^{1,2}$ Yudong Yang, ${ }^{1,2}$ \\ Shih-Hsuan Chia, ${ }^{1,2}$ Shaobo Fang,,${ }^{1,2}$ Oliver D. Mücke, ${ }^{1,2}$ Giulio Cerullo, ${ }^{5}$ and Franz X. Kärtner ${ }^{1-4}$ \\ ${ }^{1}$ Center for Free-Electron Laser Science, Deutsches Elektronen-Synchrotron DESY, Notkestraße 85, 22607 Hamburg, Germany \\ ${ }^{2}$ The Hamburg Center for Ultrafast Imaging, Luruper Chaussee 149, 22761 Hamburg, Germany \\ ${ }^{3}$ Physics Department, University of Hamburg, Luruper Chaussee 149, 22761 Hamburg, Germany \\ ${ }^{4}$ Department of Electrical Engineering and Computer Science and Research Laboratory of Electronics, \\ Massachusetts Institute of Technology, Cambridge, Massachusetts 02139, USA \\ ${ }^{5}$ IFN-CNR, Dipartimento di Fisica, Politecnico di Milano, Piazza Leonardo da Vinci 32, I-20133 Milan, Italy \\ shih-hsuan.chia@.cfel.de
}

\begin{abstract}
The relative timing jitter of a multi-mJ sub-cycle 3-channel parametric waveform synthesizer pumped by an 18-mJ cryogenically cooled Ti:sapphire laser is studied. The system aims towards multi-mJ, 2-fs, phase-stable pulses covering the $0.5-2.4 \mu \mathrm{m}$ range. OCIS codes: (320.7110) Ultrafast nonlinear optics; (190.4970) Parametric oscillators and amplifiers.
\end{abstract}

High-energy sub-cycle optical waveforms have numerous intriguing applications in attoscience and strong-field physics, e.g., in the generation of intense isolated attosecond XUV pulses [1], relativistic laser-plasma interactions and laser-driven electron acceleration [2], valence-electron wavepacket dynamics in atoms and molecules [3], and the control of sub-cycle electron transport in solids [4].

Shaping the electric field on a sub-cycle time scale requires an optical bandwidth of at least two octaves and precise control of the spectral phase over this bandwidth [5]. Supercontinuum generation in gas-filled hollow-core fiber compressors represents a rather mature technology for this purpose [3,6]. However, the scalability of gas-phase broadening schemes in terms of pulse energy and repetition rate seems to be limited by ionization losses, detrimental nonlinearities, heating-related problems and damage of materials. In contrast, parametric waveform synthesizers offer much better prospects for simultaneously scaling up both the pulse energy to the multi-mJ range and the repetition rate to tens or hundreds of $\mathrm{kHz}$, which makes them very attractive for next-generation light sources such as SLAC, SACLA, FERMI and ELI-ALPS, targeting such operation parameter ranges.

In earlier works, we already demonstrated coherent waveform synthesis between ultrabroadband 870 -nm and $2.15-\mu \mathrm{m}$ pulses based on optical parametric chirped-pulse amplification (OPCPA), resulting in a $15-\mu \mathrm{J}$ sub-cycle waveform [7], and also between two optical parametric amplifiers (OPAs) with 1-2 $\mu \mathrm{J}$ energy each [8]. More recently, we have been developing a prototype multi-mJ 3-channel parametric synthesizer for generating 2-octavewide spectra with $\sim 1.9$-fs transform-limited pulse duration [9]. In this work, we investigate and demonstrate the feasibility to perform the final synthesis step in this parallel scheme at the multi-mJ level for the first time, with important implications for the above-mentioned next-generation light sources.

In 2013, we proposed and reported first amplification results for our synthesizer pumped by a cryogenically cooled Ti:sapphire chirped-pulse amplifier (150 fs, $18 \mathrm{~mJ}, 1 \mathrm{kHz})$ [9]. We generate a common CEP-stable seed continuum $(0.5-2.5 \mu \mathrm{m})[10]$ by white-light generation in a YAG crystal pumped by the second harmonic $(1.06 \mu \mathrm{m})$ of the CEP-stable idler of a NIR OPA. The resulting continuum is then split with dichroic beam splitters [11] and seeds three OPA channels employing type-I BBO crystals, a VIS non-collinear OPA (NOPA), a NIR and an IR degenerate OPA (DOPA) channel, pumped by the fundamental wavelength pulses at $0.8 \mu \mathrm{m}$ (IR DOPA) and by its second harmonic at $0.4 \mu \mathrm{m}$ (VIS NOPA, NIR DOPA). Figure 1 shows the output spectra and pulse energies of the 3 channels obtained after 3 amplification stages each.

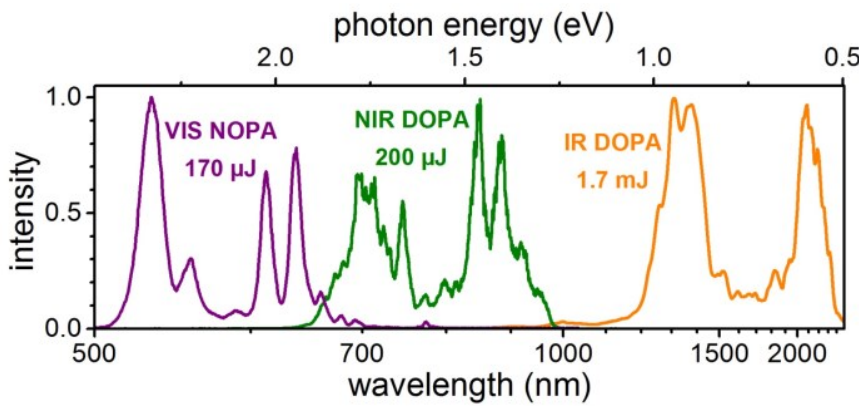

Fig. 1. Output spectra from the third stages of the VIS NOPA/NIR DOPA/IR DOPA channels. These spectra support 5.6/5.2/5.2-fs pulses corresponding to 2.9/2.1/1.1 optical cycles at $573 \mathrm{~nm} / 750 \mathrm{~nm} / 1390 \mathrm{~nm}$ center wavelengths, respectively. 
After parametric amplification, the three OPA channel outputs can individually be compressed, recombined and finally fully recompressed close to the Fourier limit inside the vacuum chamber using custom-designed precision dispersive mirrors [11]. To illustrate the precision and flexibility of our dispersion compensation scheme, we show in Fig. 2 FROG pulse-characterization results of all three second-stage outputs at the waveform synthesis point.
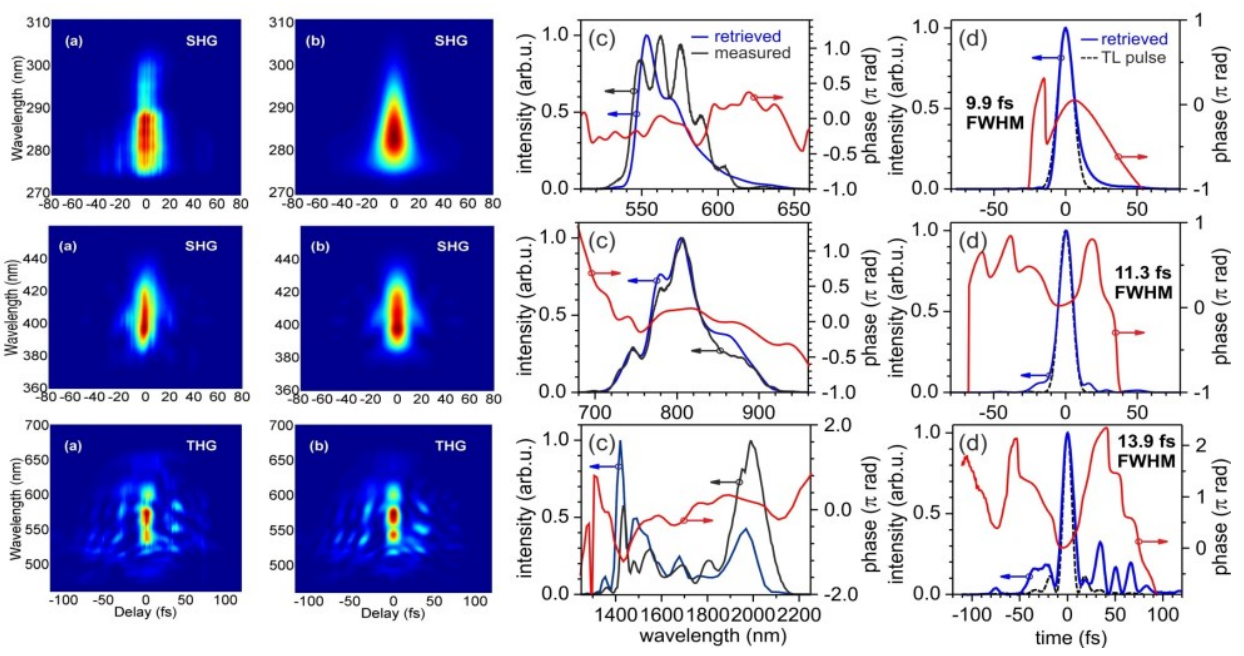

Fig. 2. FROG characterization of the second-stage OPA outputs from the three channels at the synthesis point. The VIS NOPA (top row) and NIR DOPA (middle row) are characterized by means of SHG-FROG, the IR DOPA (bottom row) using surface THG-FROG. (a) Measured and (b) retrieved FROG traces. (c) Measured spectrum, retrieved spectral intensity and phase. (d) Retrieved temporal intensity and phase profiles as well as transform-limited intensity profile. The retrieved FWHM pulse durations are indicated. No marginal correction was applied to the THG-FROG trace in the bottom row.

Up to now we have not yet performed the active feedback stabilization of the relative timing between the three synthesized channels with sub-cycle precision, which is a prerequisite for coherent waveform synthesis [5]. In previous works by Leitenstorfer's group [12], attosecond-level relative timing jitter between two channels of a nJlevel waveform synthesizer based on erbium-doped fiber technology was demonstrated. Our own earlier experiments $[7,8]$ demonstrated coherent waveform synthesis on the $\mu \mathrm{J}$ level already. In this paper, we present the first detailed investigation of the relative timing jitter properties of a scaled-up multi-mJ sub-cycle waveform synthesizer, which has much longer beam path (several tens of meters). Our results reveal a high passive timing stability between the synthesized channels, which corroborates the feasibility to actually perform the coherent synthesis after applying straightforward improvements in the stabilization of the beam path.

We combined spatially and temporally the three OPA pulses after the third amplification stages (VIS NOPA3, NIR DOPA3, IR DOPA3) on custom-designed dichroic beam splitters [11], which have a $\sim 5 \%$ leakage composed of the combined OPA pulses collinear with each other. This leakage is used for the balanced optical cross-correlator (BOC) setups, analogous to the ones described in $[7,8]$. We first implemented the BOC for VIS NOPA3 and IR DOPA3. The BOC consists of two identical cross-correlators, in which the 580-nm component from the VIS NOPA3 and the 1550-nm component from IR DOPA3 generate the sum frequency at $420 \mathrm{~nm}$, in two 1-mm-thick type-I BBO crystals. A 3-mm-thick glass plate is inserted in one of the cross correlators, to reverse the time order of the two pulses. The sum-frequency signals are filtered within a 10-nm optical bandwidth in front of the home-made detection system discussed below. The IR pulses are used as the reference, and we use a piezo-transducer (PZT) actuated delay stage in the VIS channel to compensate for the relative timing jitter.

The balanced photodetector uses a new home-built design. The charge deposited onto the photodiode (PD) upon illumination is integrated completely rather than sampling a fraction of it by sample-and-hold as in previous BOC implementations [7,8]. It also omits the need for a low-jitter trigger signal, high-speed precision electronics or data acquisition devices. This approach improves the signal-to-noise ratio and simultaneously allows single-shot data acquisition. The analog frontend is kept as basic as possible. The signal of each PD is individually integrated and converted into digital. The balancing of the two PDs is done via software running on a microcontroller/FPGA, which controls the device. This also offers the possibility to transfer the data (PD1, PD2, difference) to a PC or monitor the data online in time and frequency domain. Moreover, a PID control loop can directly be implemented to run on the unit to feedback to the PZT to compensate for the measured timing jitter.

In Fig. 3, we report the first characterization data of the natural (i.e., passive, unstabilized) timing jitter between the VIS NOPA3 and IR DOPA3 outputs in our multi-mJ waveform synthesizer. The typical S-shaped BOC signal displayed in Fig. 3(a) features a $\sim 100$-fs wide linear region, which can directly be used for feedback stabilization of the relative timing. For the moment, to investigate the noise properties and to evaluate the overall passive timing stability of the system, we used the following experimental procedure: we injected a known sinusoidal signal at 33.3 $\mathrm{Hz}$, with 1-V peak-to-peak amplitude driving the PZT, used for adjusting the relative timing. From the specifications 
of the PZT (Physik Instrumente 601.1SL), we calibrated the rms of this injected sinusoidal signal to be $\sim 2.4$ fs. Afterwards, by comparing the red area in Fig. 3(b) corresponding to the known injected noise to the blue area corresponding to the passive timing jitter, we could evaluate the overall timing jitter to be $\sim 4.3 \mathrm{fs} \mathrm{rms}$. Note that the PZT-based feedback stabilization can easily eliminate the low-frequency timing jitter.
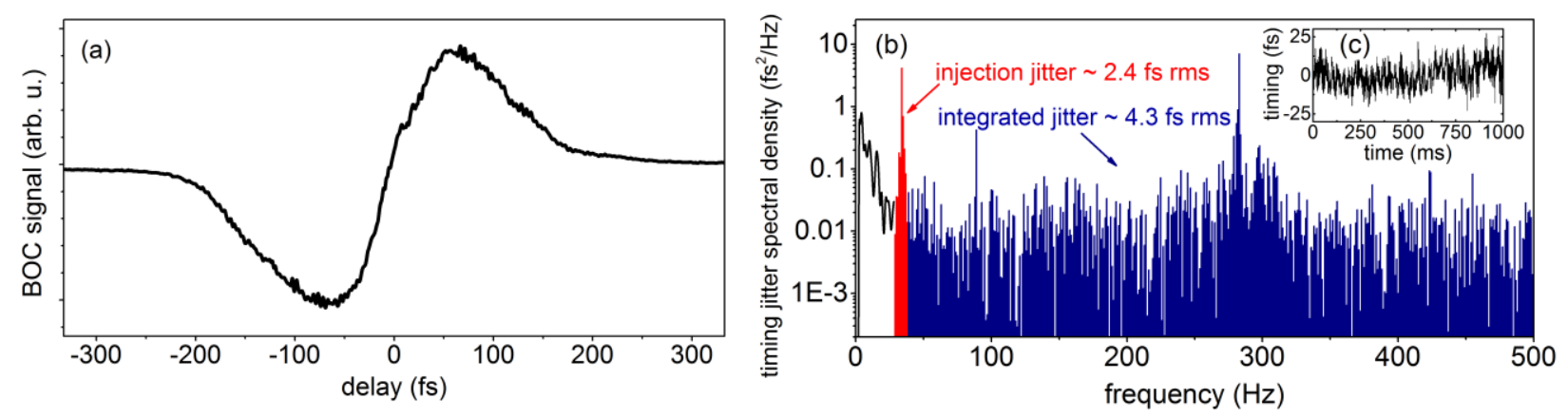

Fig. 3. (a) BOC S-curve for VIS NOPA3 and IR DOPA3, exhibiting a $\sim 100$-fs wide linear region useful for feedback stabilization. (b) Natural timing jitter spectral density between the two channels measured by the same BOC. The red area corresponds to the injected sinusoidal test signal with $\sim 2.4$-fs rms jitter at $33.3 \mathrm{~Hz}$, while the blue area corresponds to the natural (i.e., passive, unstabilized) timing jitter. By comparison with the injected signal peak, the natural timing jitter is evaluated to be only $\sim 4.3 \mathrm{fs}$ rms. The inset (c) shows the same BOC signal in the time domain with the clear modulation from the injected $33.3-\mathrm{Hz}$ test signal.

The main contribution to the timing jitter could be traced back to an excessively loud turbo vacuum pump directly attached to the cryo Ti:sapphire booster amplifier driving our synthesizer. Close to this pump, we measured a $6 \mathrm{~dB}$ higher acoustic noise power as compared to other even bigger turbo pumps in our lab. The $\sim 85-\mathrm{Hz}$ spike observed in Fig. 3(b) corresponds to the down-sampled vibrations of this turbo pump (rotating at $1085 \mathrm{~Hz}$ ). The broad noise band from 250 to $330 \mathrm{~Hz}$ originates from mechanical vibrations excited by this excessive noise. We observed that the noise band amplitude and its shape vary with the rotation speed of the turbo pump. We are convinced that replacement of the noisy pump by a much quieter one and an improved vibration isolation will strongly suppress the high-frequency timing jitter permitting a sub-cycle timing lock.

Apart from the turbo pump noise issue, the observed surprisingly low $\sim 4.3-\mathrm{fs}$ rms timing jitter seems a direct consequence of our parallel synthesizer architecture, in which all 3 channels are seeded by a common CEP-stable supercontinuum generated in a YAG plate $[9,10]$. On a second-time-scale, the passive stability of our setup is sufficient to guarantee already a few-fs timing stability. In contrast, if the channels were seeded by different whitelight supercontinua created from separate pulses with uncorrelated pulse-energy fluctuations, a substantial timing jitter [13] between the synthesizer channels could occur.

In summary, this work shows that the rms timing jitter between parallel OPA channels in a multi-mJ sub-cycle waveform synthesizer is already at the level of 1-2 optical cycles without any active stabilization. Elimination of the major noise sources, here the vibration and acoustics of a noisy turbo pump, and using state-of-the-art electronic feedback should enable locking of the different sub-pulses well below a tenth if not a hundredth of an optical cycle. The synthesizer will be a versatile tool for controlling strong-field interactions in atoms, molecules and solids and for attosecond pump-probe spectroscopy employing ultrashort pulses in the VIS/IR and XUV/soft-X-ray regions.

\section{References:}

[1] E. J. Takahashi et al., Nat. Commun. 4:2691 (2013); E. J. Takahashi et al., Phys. Rev. Lett. 104, 233901 (2010).

[2] L. Veisz et al., CLEO Pacific Rim 2013, paper TuD2-3; L.-J. Wong et al., Opt. Lett. 36, 957 (2011).

[3] A. Wirth et al., Science 334, 195 (2011). Also see: M. T. Hassan et al., Rev. Sci. Instrum. 83, 111301 (2012); T. T. Luu et al., QELS 2013, paper QF1C.6.

[4] O. D. Mücke, Phys. Rev. B 84, 081202(R) (2011); S. Ghimire et al., Nature Phys. 7, 138 (2011); A. Schiffrin et al., Nature 493, 70 (2013).

[5] C. Manzoni et al. Laser \& Photonics Rev. in press (2014).

[6] S. Fang et al., HILAS 2014, paper HW1C.2; S. Fang et al., Ultrafast Phenomena 2014, paper 09.Wed.P3.60.

[7] S.-W. Huang et al., Nature Photonics 5, 475 (2011); S.-W. Huang et al., J. Phys. B 45, 074009 (2012).

[8] C. Manzoni et al., Opt. Lett. 37, 1880 (2012).

[9] G. Cirmi et al., Ultrafast Optics 2013 (UFO IX), paper We3.3; G. M. Rossi et al., CLEO 2014, paper SF1E.3.

[10] G. Cerullo et al., Laser \& Photonics Rev. 5, 323 (2011).

[11] S.-H. Chia et al., Optica in press (2014); S.-H. Chia et al., ASSL 2014, paper ATu3A.2.

[12] F. Adler et al., Opt. Lett. 32, 3504 (2007); G. Krauss et al., Nature Photonics 4, 33 (2011).

[13] C. Manzoni et al., Phys. Rev. A 79, 033818 (2009). 